\title{
USABILITY EVALUATION OF A MULTIMEDIA ARCHIVE: B@BELE
}
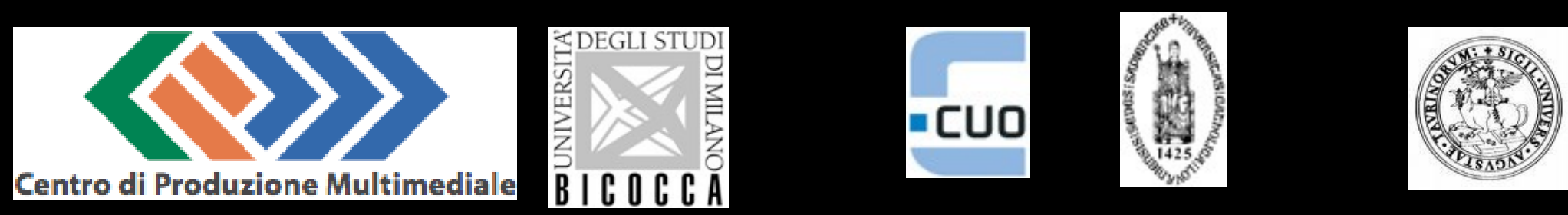


\section{WHY A USABILITY STUDY}

0

$\circ$
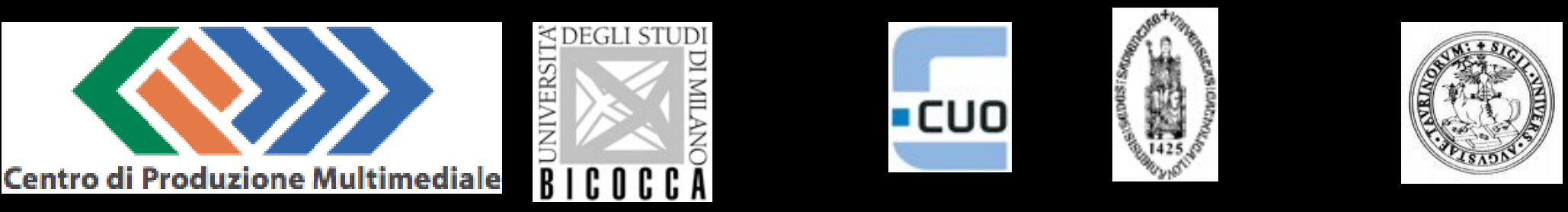


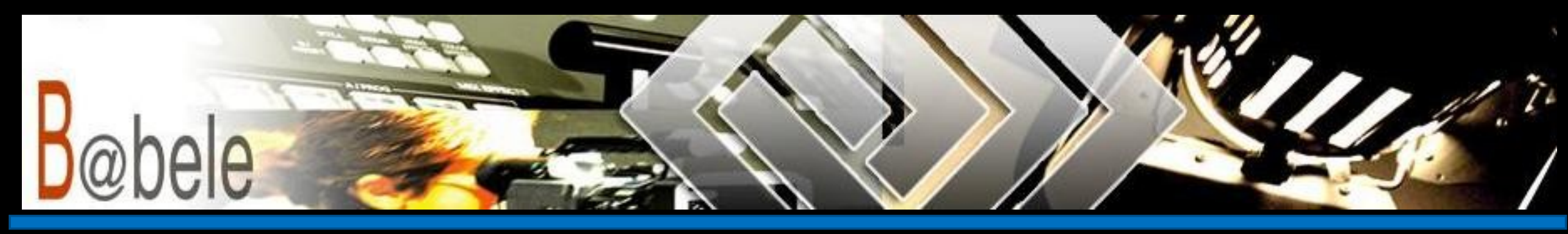

0

0
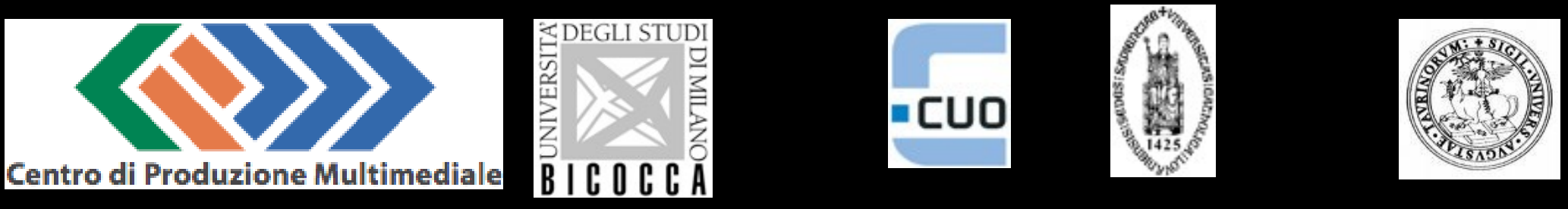


\section{Benvenuto in B@bele, CPM Digital Repository}

B@bele è l'archivio digitale del Centro di Produzione Multimediale dell'Università di Milano-Bicocca. Contiene collezioni di materiale multimediale curate da docenti, ricercatori e uffici amministrativi dell'università, relative a progetti di ricerca, tesi di dottorato, materiale didattico e informativo, eventi organizzati all'interno dell'ateneo. Alcune collezioni sono di pubblico accesso, altre

richiedono una registrazione al sito o sono scaricabili a richiesta. E' possibile navigare attraverso l'indice delle collezioni o utilizzando il menu laterale, o effettuare una ricerca inserendo una parola chiave nel box di ricerca.

\section{Ricerca in DSpace}

Inserisci un testo nel box sottostante per effettuare ricerche in DSpace.

\section{Vai}

\section{Comunità in DSpace}

Selezionare una Sottocomunità per visualizzare le sue collezioni.

- ARCHIVIO GM - VERSIONE DEMO

- ARCHIVIO MULTIMEDIALE di ATENEO

- CPM - CENTRO DI PRODUZIONE MULTIMEDIALE

- DOTTORATO qUaSi

- NOMADIS

- ORIENTARCHIVIO

- test usabilitá
Cerca in DSpace

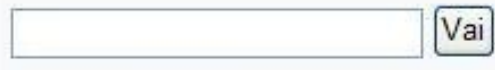

Ricerca Avanzata

\section{Ricerca}

\section{Tutto DSpace}

Comunità \&. Collezioni Data di pubblicazione Autori

Titoli

Soggetti 


\section{OBJ ECTIVES OF THE EVALUATION}

\section{EVALUATE B@BELE MAIN FUNCTIONALITIES}

\section{SEARCH}

The functionality of the search interface both in its simple and advanced version and navigation within each community and collection.

\section{SELF-ARCHIVE}

The functionality of the submission interface, i.e., the process of inserting items and documents with reference to the main options during workflow. management both in their submission phase and in their modification phase 


\section{OBJ ECTIVES OF THE EVALUATION/2}

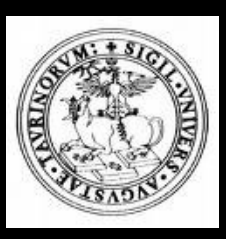




\section{SELECTED TEST SUBJ ECTS}

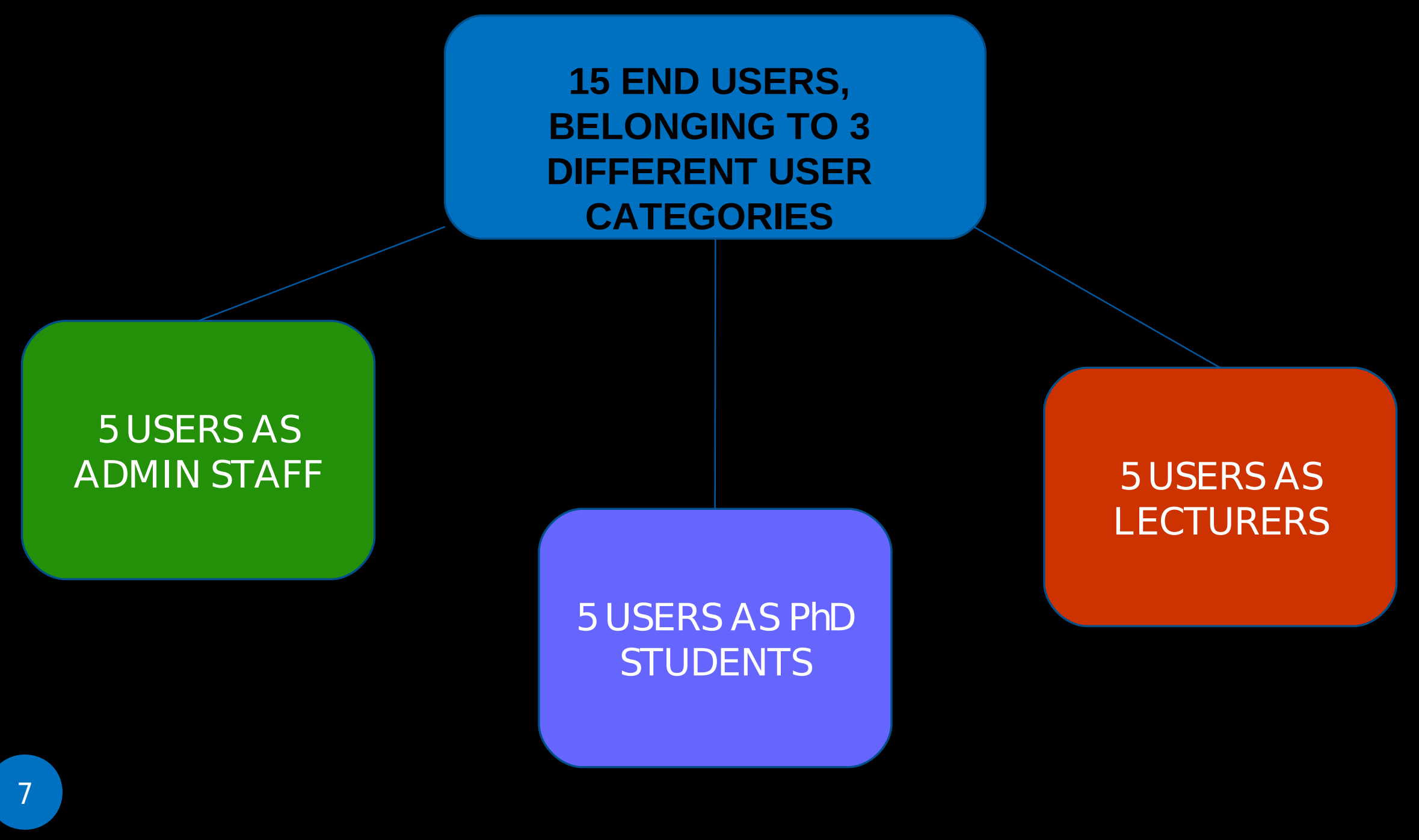




\section{METHODOLOGY}

0

O

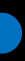
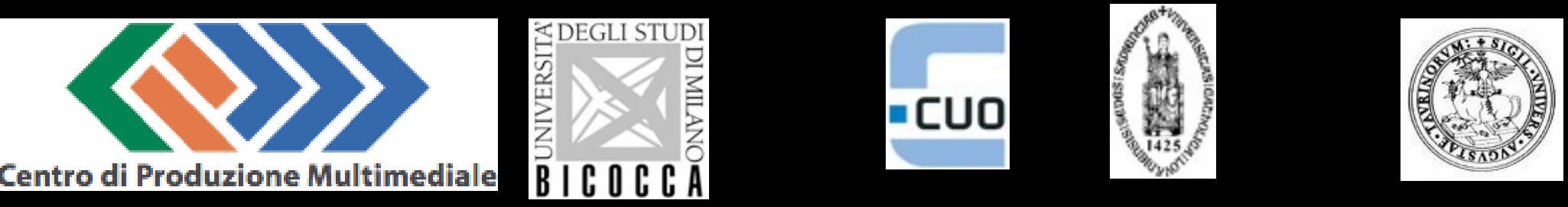


\section{RESULTS: TERMINOLOGY}

O

O

O
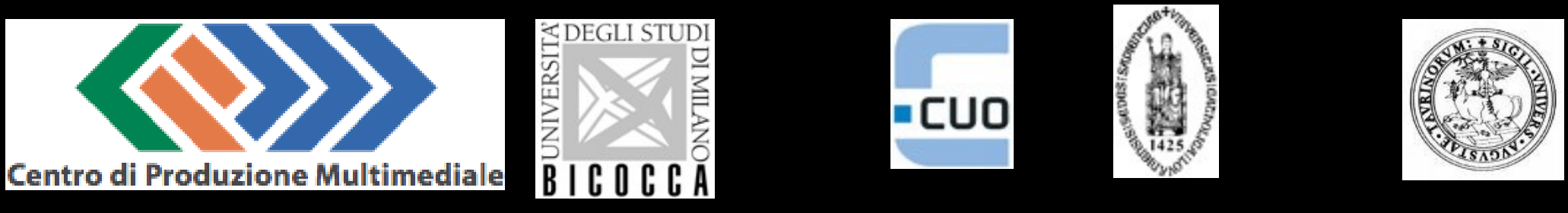


\section{RESULTS: TERMINOLOGY/2}

O

0
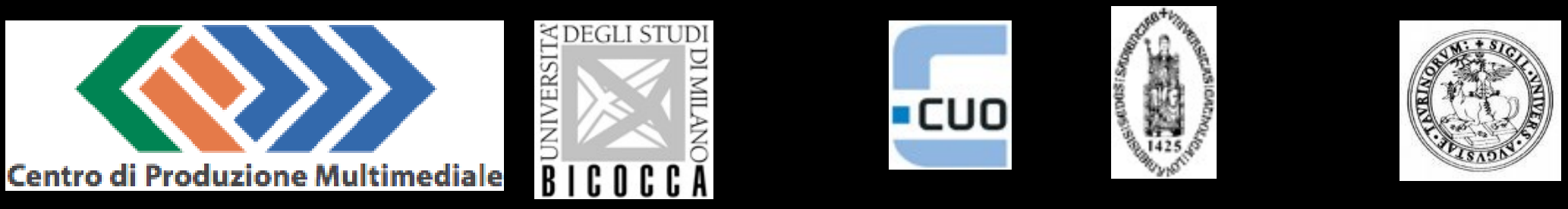


\section{RESULTS: BROWSING IN COMMUNITIES AND COLLECTIONS}

\section{Comunità in DSpace}

Selezionare una Sottocomunità per visualizzare le sue collezioni.

- ARCHIVIO GM - VERSIONE DEMO

- Manifestazioni e manifestanti

- Immigrant Rights

- Quotidiani e riviste

- Corriere della sera

- The New York Times

- Strategie di sopravvivenza

- Bangladesh

- Brasile

- Trasformazioni metropolitane

- Mappe tematiche

- Origine della citta'

- ARCHIVIo MULTIMEDiALE Di ATENEO

- Campus - Photogallery

- Edifici e strutture

- Vita nel Campus

- Eventi 


\section{COMMUNTIES AND COLLECTIONS/2}

0

0

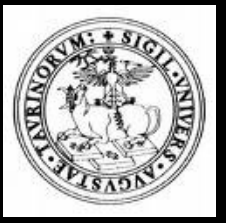




\section{RESULTS: UPLOAD}

O

Immissioni \& Workflow

Workflow

Di seguito gli items in attesa di approvazione. Vi sono due tipologie di incarichi, una per quelli che hai accettato e un'altra per quelli ancora non assegnati.

Tuoi incarichi

Incarichi

Item

Collezioni

Utente

Non hai incarichi assegnati

Incarichi completi

\begin{tabular}{|llll}
\multicolumn{1}{c}{ Incarichi } & \multicolumn{1}{c}{ Item } & \multicolumn{1}{c}{ Collezioni } & \multicolumn{1}{c}{ Utente } \\
$\begin{array}{llll}\text { In attesa dell'attenzione del } \\
\text { revisore }\end{array}$ & $\begin{array}{l}\text { Ricerca sul } \\
\text { campo }\end{array}$ & $\begin{array}{l}\text { Test } \\
\text { usabilità }\end{array}$ & $\begin{array}{l}\text { email: Dspace } \\
\text { Usabilità }\end{array}$ \\
\hline Accetta gli incarichi selezionati & & & \\
\hline
\end{tabular}

Immissioni non terminate

Queste sono le immissioni incomplete. Potete anche iniziare una nuova immissione.

\begin{tabular}{|c|c|c|c|}
\hline & Titolo & Collezione & Utente \\
\hline$\square$ & Ricerca sul campo & Test usabilità & email: Dspace Usabilità \\
\hline$\square$ & ricerca sul campo & Test usabilità & email: Dspace Usabilità \\
\hline
\end{tabular}




\section{RESULTS: WORKFLOW}

Immissioni \& Workflow

Workflow

Di seguito gli items in attesa di approvazione. Vi sono due tipologie di incarichi, una per quelli che hai accettato e un'altra per quelli ancora non assegnati.

Tuoi incarichi

Incarichi

Item

Collezioni

Utente

Non hai incarichi assegnati

Incarichi completi

\begin{tabular}{|llll}
\multicolumn{1}{c}{ Incarichi } & Item & Collezioni & Utente \\
$\begin{array}{llll}\text { In attesa dell'attenzione del } \\
\text { revisore }\end{array}$ & $\begin{array}{l}\text { Ricerca sul } \\
\text { campo }\end{array}$ & $\begin{array}{l}\text { Test } \\
\text { usabilità }\end{array}$ & $\begin{array}{l}\text { email: Dspace } \\
\text { Usabilità }\end{array}$ \\
\hline Accetta gli incarichi selezionati & & & \\
\hline
\end{tabular}

Immissioni non terminate

Queste sono le immissioni incomplete. Potete anche iniziare una nuova immissione.

\begin{tabular}{llll} 
& \multicolumn{1}{c}{ Titolo } & Collezione & Utente \\
$\square$ & Ricerca sul campo & Test usabilità & email: Dspace Usabilità \\
$\square$ & ricerca sul campo & Test usabilità & email: Dspace Usabilità
\end{tabular}




\section{GUIDELINES: GENERAL}

0

0
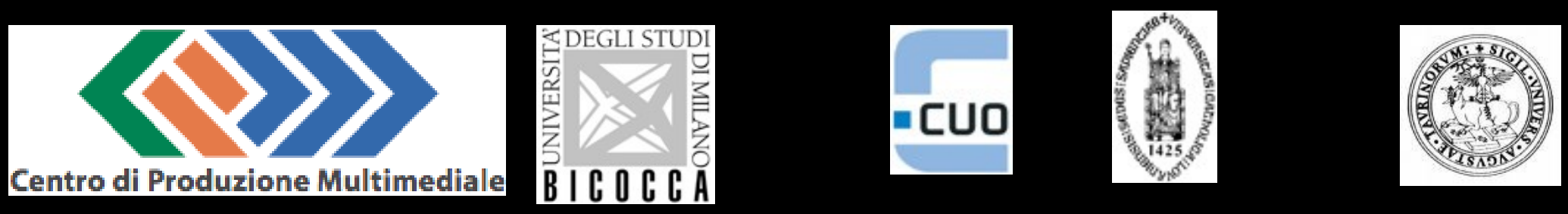


\section{GUIDELINES: TERMINOLOGY}

0

O

O
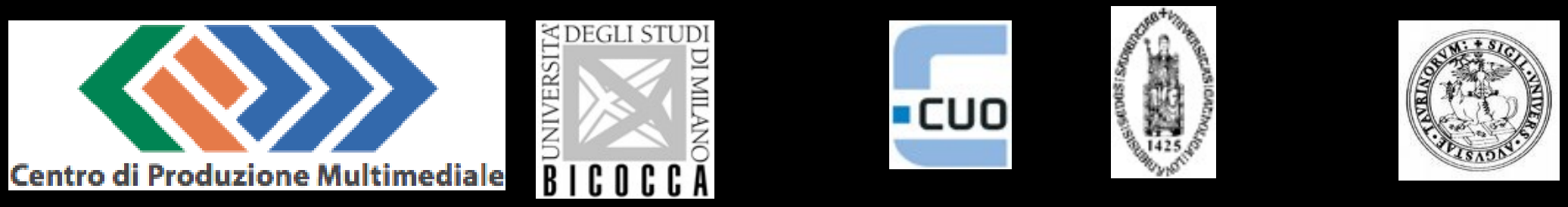


\section{GUIDELINES: NAVIGATION}

0

O

O

O
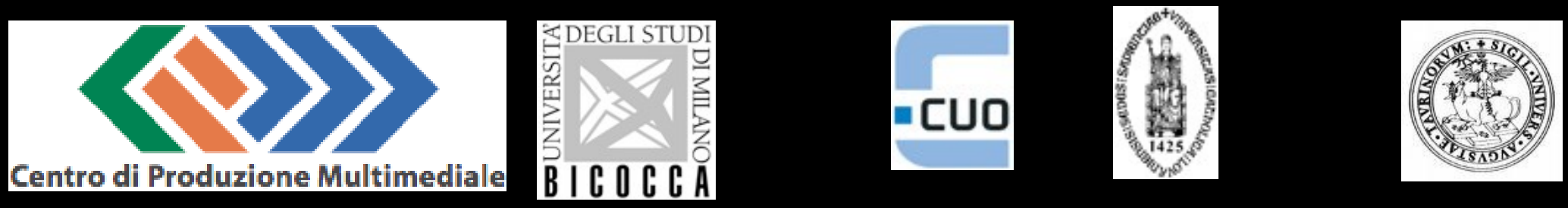


\section{GUIDELINES: NAVIGATION/2}

0

O
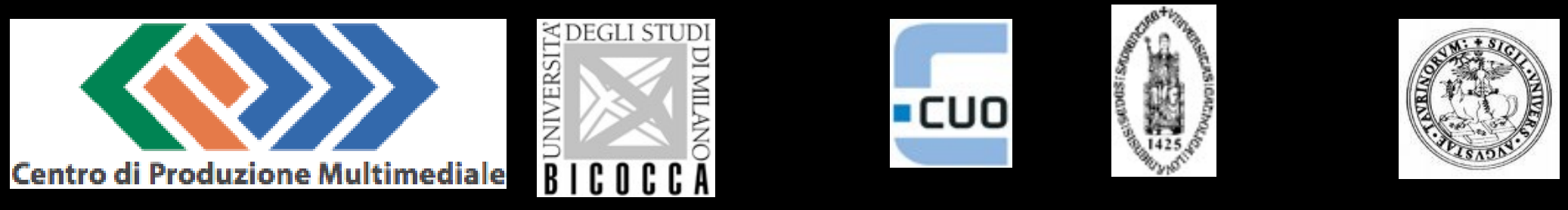


\section{GUIDELINES: SUBMISSION}

0

O
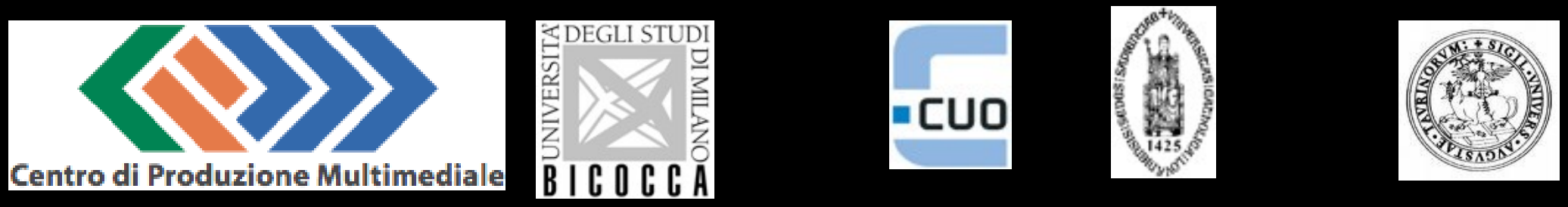


\section{GUIDELINES: WORKFLOW}

0

O
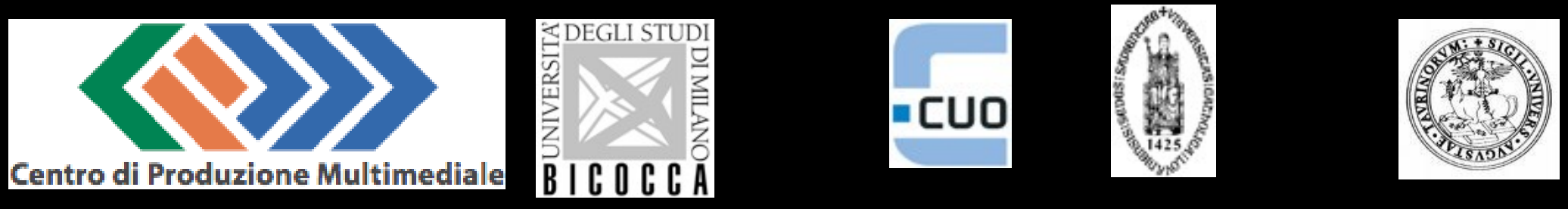


\section{CONCLUSION}

0

O

O

0

O
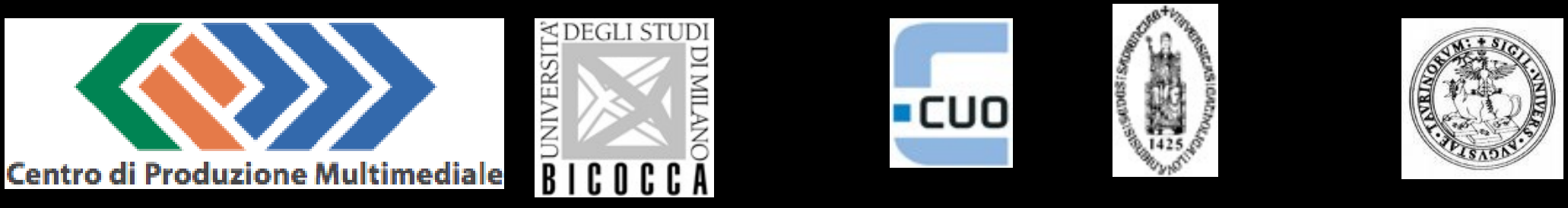


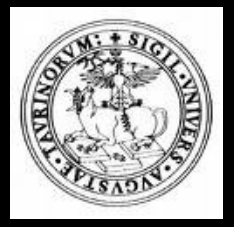

